\title{
A Retrospective Cross-Sectional Study on the Prevalence of E-cigarette Use Among College Students
}

\author{
Rebecca D. Jones $^{1} \cdot$ Matthew Asare ${ }^{1,2}$ (D) $\cdot$ Beth Lanning ${ }^{1}$ \\ Published online: 26 June 2020 \\ ○) Springer Science+Business Media, LLC, part of Springer Nature 2020
}

\begin{abstract}
E-cigarette use among young adults is a major public health concern. Approximately $17.7-40 \%$ of college students have tried or are currently using e-cigarettes. While a few studies have examined e-cigarette use among youth, opportunity exists to understand psychosocial factors that influence college students' e-cigarette behavior. The main purpose of this study is to examine the associations between the constructs of self-efficacy, knowledge, depression and anxiety symptoms, and e-cigarette use among college students. A retrospective cross-sectional survey (20-items) design was used for data collection. Bivariate analysis and analysis of variance (ANOVA) were used to evaluate associations between the independent variables and the dependent variable. A total of 872 college students between the ages of 18 and 25 completed the survey in Qualtrics. A significant association between gender and frequency of e-cigarette use was found $\left(\chi^{2}=22.94, p<.001\right)$. ANOVA results showed significant relationships between knowledge $[\mathrm{F}(3,808)=9.01, \mathrm{p}<0.001]$, self-efficacy $[\mathrm{F}(3,808)=4.85, \mathrm{p}<0.01]$, depression $[\mathrm{F}(3,808)=8.31, \mathrm{p}<.05]$, and e-cigarette use. Post hoc analysis revealed students who never used e-cigarettes scored higher on knowledge and self-efficacy than students who used every day, somedays or rarely, indicating never-users have higher knowledge of negative effects associated with e-cigarette use and have higher self-confidence than e-cigarette users. The study's findings highlight that modifiable factors such as knowledge about harmful effects of e-cigarettes and self-confidence are associated with low e-cigarette use. Interventions could be designed to target these modifiable factors.
\end{abstract}

Keywords Vaping $\cdot$ E-cigarette $\cdot$ Self-efficacy $\cdot$ College students $\cdot$ Depression $\cdot$ Anxiety

\section{Introduction}

Tobacco use has been a health concern for years. Recently, with combustible cigarette use on the decline, a new tobacco-related concern has arisen in the form of e-cigarettes. In 2016 the Surgeon General officially declared e-cigarette use among the United States (U.S.) youth as a major public health issue [32]. E-cigarettes come in many different varieties, sizes, and flavors [32]. E-cigarette devices are composed of a battery, a reservoir for e-liquid typically containing nicotine, a heating element, and a mouthpiece [32]. One of the defining characteristics of the recent generation

Matthew Asare

matt_asare@baylor.edu

1 Department of Public Health, Baylor University, Waco, TX, USA

2 Department of Public Health, Robbins College of Health and Human Sciences, Baylor University, One Bear Place \#97343, Waco, TX, USA of devices is that they contain larger batteries capable of heating the e-liquid to a higher temperature, allowing the device to release more nicotine, form additional toxicants, and create larger clouds of particulate matter [19].

\section{E-cigarette Use Among College Students}

The U.S. Centers for Disease Control and Prevention (CDC) and the Food and Drug Administration (FDA) showed in a National Youth Tobacco Survey (NYTS) that e-cigarettes have been the most commonly used tobacco product among U.S. youth since 2014 . Approximately $17.7-40 \%$ of college students have tried or are currently using e-cigarettes $[12,15,21]$ and $60 \%$ of college students have been offered an e-cigarette at least once [10, 33]. Of college e-cigarette users, $40 \%$ had not previously smoked combustible cigarettes [28] and e-cigarette use is positively associated with willingness to try combustible cigarettes in the future [9]. These findings indicate that e-cigarette use is creating a new 
population of individuals vulnerable to developing a nicotine addiction.

\section{Health Effects of E-cigarette Use}

There are many negative health effects associated with youth tobacco consumption, the most predominant of which is a high risk of addiction. According to the National Center on Addiction and Substance Abuse, the risk of nicotine addiction increases with earlier age of first use [31]. Nicotine exposure during brain development, which continues until the mid-twenties, is associated with decreased attention span later in life [11] and learning disabilities in adulthood [13].

The negative health effects of e-cigarette use are related to the chemical components within e-liquids. Detectable levels of more than 115 volatile compounds have been found in a single puff of an e-cigarette [31]. Many of the potentially toxic chemicals found in the aerosol were not present in the e-liquid solution suggesting that the aerosolization process itself might increase the risks associated with e-cigarette use [31]. The documented acute effects of e-cigarette use include increased plasma nicotine, heart rate, and carbon monoxide concentration [31]. Other adverse effects include respiratory distress, bronchitis, impaired vascular function, cell damage that can lead to oral disease, and links to cardiovascular disease and cancer [31].

E-cigarette use may also lead to an increased risk of respiratory diseases. Covid-19 is a respiratory disease caused by the virus severe acute respiratory syndrome coronavirus 2 (SARS-CoV-2) [4]. Due to the lung damage caused by sustained e-cigarette use, smokers could be at a higher risk of infection and severe presentation of Covid-19. Research has shown that smoking, including e-cigarette use, can lead to an upregulation of the angiotensin-converting enzyme-2 (ACE2) receptor, the known receptor for the coronavirus family, including SARS-CoV-2 [4]. Attachment of the virus to ACE2 receptors on the cell surface protects the virus from immune surveillance mechanisms, allowing the virus to remain unbothered in the host for long periods of times thus making the host susceptible to future infections as well as increasing the spread of the virus [4]. This upregulation of ACE2 receptors may put smokers at an increased risk for Covid-19.

\section{Mental Health and E-cigarette Use}

High rates of e-cigarette use have been linked to an increase in depressive symptoms among young adults [22]. Former e-cigarette users have 1.6 times higher odds of reporting a history of depression than those who never used and current e-cigarette users have a 2.10 times higher odds [26].
Higher odds of depression have also been observed with increased frequency of use among current e-cigarette users compared with never users [26]. Reports indicate that a single day of nicotine exposure during adolescence is sufficient to precipitate a negative emotional state rendering the individual increasingly vulnerable to the adverse effects of stress and subsequent depression in adulthood [17]. Furthermore, Bandiera et al. (2017) found that elevated depression symptoms predicted future e-cigarette use. A bi-directional relationship between depression symptoms and e-cigarette use has been suggested, implying that depressed individuals may smoke as a form of self-medication and that smoking may lead to increased depressive symptoms $[2,7]$. The bidirectionality of the relationship between e-cigarette use and depression is further supported by Chaiton et al. [8], whose study suggests that the pathways from depressive symptoms to smoking differ from the pathways that relate smoking to depressive symptom onset.

While previous studies have indicated a relationship between depression and e-cigarette use [2, 17, 22], few studies have focused on anxiety and e-cigarette use. A higher correlation has been seen between individuals suffering from both depression and anxiety or individuals suffering solely from anxiety and smoking combustible cigarettes than those suffering from depression alone [25]. Smoking combustible cigarettes has also been associated with an increased risk of mood and anxiety disorders [24]. Mild to severe mental distress, as seen in individuals suffering from anxiety and depression, has been shown to produce a negative impact on academic performance [3].

\section{E-cigarette Marketing Strategies}

E-cigarette manufacturers target college students through the use of the internet and social media as the primary source for e-cigarette advertisements, many of which perpetuate misinformation on the risks associated with e-cigarette use. An analysis of e-cigarette videos on YouTube found that $85 \%$ of e-cigarette promotional videos were produced directly by e-cigarette manufactures and $94 \%$ were "pro" e-cigarette use [23]. Ninety-five percent of e-cigarette retailer websites make explicit or implicit health-related claims, $64 \%$ had smoking cessation-related claims, and $22 \%$ featured doctors [14]. These advertisements influence perceptions of e-cigarettes leading users and nonusers alike to cite e-cigarette use as a healthier alternative to smoking combustible cigarettes and reporting that they believe e-cigarettes contain fewer chemicals, less nicotine, and less smoke than their combustible counterparts [5]. A commonly cited motivation among college students for using e-cigarettes is the belief that it is a healthy alternative to combustible cigarettes $[16,21]$. 


\section{The Present Study}

While a few studies have examined e-cigarette use among youth, opportunity exists to understand psychosocial factors that influence college students' e-cigarette behavior. The purpose of this study was to explore the relationship between five measures of interest-knowledge of negative health effects from e-cigarette use, self-efficacy, depression and anxiety symptoms, and academic performanceand e-cigarette use behavior among college students.

Knowledge and depression were selected as measures of interest due to previous research implicating these as influential on e-cigarette use. While previous studies have looked at high self-efficacy and intention to quit e-cigarette use [7, 27], this study was designed to assess the relationship between low self-efficacy and frequency of e-cigarette use. Previous studies have found a relationship between smoking combustible cigarettes and anxiety symptoms [25] however, studies assessing the relationship between e-cigarette use and anxiety are lacking. Additionally, it has been shown that mental distress, as seen in individuals suffering from depression or anxiety symptoms, can have a negative impact on academic performance [3]. Therefore, this study aims to assess the relationship between academic performance and e-cigarette use.

\section{Methods}

\section{Design}

A retrospective cross-sectional survey design was used to collect data from college students between the ages of 18 and 25. Participants were asked to complete an online Qualtrics survey on vaping behavior. Data was collected between August 2019 and January 2020. Prospective participants were assured that their participation was completely voluntary, anonymous, and confidential and that there would be no penalty for declining to participate, discontinuing at any time, or omitting answers to any questions.

\section{Informed Consent}

Before beginning the survey, participants were asked to read a consent form. We indidicated in the consent form that by clicking "next" to continue to the fill out the survey, participants indicated their consent to participate. The study was approved by the university institutional review board.

The 20-item survey included questions related to demographics, frequency of e-cigarette use, knowledge, and perceptions surrounding e-cigarette use, anxiety and depression symptoms, academic performance, and self-efficacy.

\section{Outcome Measure}

E-cigarette use frequency was assessed using the question "How often do you vape?" with possible responses of "every day", "somedays", "rarely", or "not at all".

\section{Independent Variables}

The constructs selected as independent variables included knowledge of negative effects associated with e-cigarette use, self-efficacy, anxiety and depression symptoms, and academic performance. Knowledge and perceptions surrounding E-cigarette use were assessed using eight Likert scale questions. Questions included "Vaping is harmful to your health", "Vaping may lead to future use of regular cigarettes", "Vaping is a public health concern", "Vaping should be regulated like other tobacco products", and "Vaping should be regulated in work or public places". The response options were a 5-point Likert scale (Strongly Agree, Agree, Neither Agree nor Disagree, Disagree, and Strongly Disagree). Three questions were reverse coded including "Vaping is safer than regular cigarette use", "Vaping is less addictive than regular cigarette use" and "Vaping is a helpful aid for smoking cessation". A total knowledge score was created for each participant by adding the scores of each question.

Self-efficacy was assessed using the General Self-Efficacy Scale (GSE), a ten-question Likert scale survey with a score range between 10 and 40 [29]. The GSE has a Cronbach's Alpha between 0.76 and 0.90 [29]. Academic performance was evaluated using self-reported overall Grade Point Average (GPA). This method of assessment for academic performance was selected because overall GPA reliability is found to be 0.94 [1]. Self-reports of GPA and GPAs reported from the registrar have been found to correlate as high as 0.97 [6].

Anxiety symptomology was assessed using the Generalized Anxiety Disorder scale (GAD-7), a seven-question assessment with a 4-point Likert scale response option (not at all, several days, more than half of the days and nearly every day). The possible score range was 0-28 [30]. The GAD-7 has a Cronbach's Alpha of 0.89 [30].

Depression symptomology was assessed using the Patient Health Questionnaire (PHQ-9), a nine-item survey with a 4-point Likert scale response option (not at all, several days, more than half of the days and nearly every day). The possible score range was 0-36 [20]. The PHQ-9 has a Cronbach's Alpha of 0.89 [20]. 


\section{Statistical Analysis}

Descriptive statistics summarizing the characteristics of the sample were provided. The extent to which knowledge, selfefficacy, depression, anxiety, GPA, and gender effect e-cigarette use frequency was assessed using bivariate analysis. One-way analysis of variance (ANOVA) was used to compare means differences among e-cigarette users on the independent variables. All analysis was conducted using IBM SPSS Statistics 26. Significance was set at P-value $<0.05$.

\section{Results}

\section{Descriptive Characteristics}

Of the eligible students, 873 participated in the survey. Sixty-two responses were eliminated for missing data on both the dependent and independent variables. Excluding missing data from the final analysis is consistent with the literature [18]. The sample population was primarily females $(61.7 \%)$ in their senior year of college $(52.1 \%)$. Over half of the respondents were non-Hispanic white $(62.1 \%)$, with $13.5 \%$ of respondents identifying as Hispanic/Latino, $6.8 \%$ as African American, $0.5 \%$ as Native American, $12.1 \%$ as Asian or Pacific Islander, and 5\% as "other". Descriptive characteristics of e-cigarette frequency are reported in Table 1. Nearly one-fourth $(24.8 \%)$ of the participants reported e-cigarette use with $11.5 \%$ reporting rare use, $6.3 \%$ use on somedays and, $7 \%$ reporting everyday e-cigarette use. A chi-squared analysis showed a significant association between gender and frequency of e-cigarette use $\left(\chi^{2}=22.94\right.$, $\mathrm{p}<.001)$.

\section{Main Results}

Table 2 depicts the mean scores of each independent variable for all demographic characteristics. Females' mean score $(\mathrm{M}=32.9, \mathrm{SD}=4.50)$ for knowledge was significantly higher than that of males $(M=28.71, S D=4.96)$. Many females reported significantly higher anxiety symptoms $(\mathrm{M}=11.42$, SD 4.37) than males $(\mathrm{M}=10.41,4.33)$.

A one-way between-group ANOVA was conducted to compare the effect of knowledge, self-efficacy, depression, anxiety, and GPA on e-cigarette use (see Table 3). ANOVA analyses showed a significant effect of knowledge on the four groups (everyday, somedays, rarely, and never) users $[\mathrm{F}(3,808)=9.01, \mathrm{p}<0.001]$. Post hoc comparisons using the Hochberg's GT2 test indicated that never e-cigarette users' mean scores for knowledge on e-cigarette $(\mathrm{M}=28.66$, $\mathrm{SD}=3.10$ ) was significantly higher than the everyday users $(M=26.62, S D=4.77)$ and someday users $(M=27.33$, $\mathrm{SD}=4.2$ ). Self-efficacy has significant impact on college student's e-cigarette use $[\mathrm{F}(3,808)=4.85, \mathrm{p}<0.01]$. Post hoc comparisons using the Hochberg's GT2 test indicated that never users mean score for self-efficacy on e-cigarette $(\mathrm{M}=31.85, \mathrm{SD}=5.04)$ was significantly higher than the everyday users $(\mathrm{M}=29.01$., $\mathrm{SD}=8.87)$ and rarely users $(\mathrm{M}=31.67, \mathrm{SD}=5.73)$.

Depression symptomology has significant impact on college student's e-cigarette use frequency $[\mathrm{F}(3,808)=8.31$, $\mathrm{p}<.05]$. Post hoc comparisons using the Hochberg's GT2 indicated that never users mean score for depression
Table 1 Frequency of E-cigarette use by demographic characteristics $(n=811)$

\begin{tabular}{lllllll}
\hline Characteristic & $\mathrm{n}$ & Everyday & Somedays & Rarely & Never & P-value \\
\hline Gender & & & & & & $<.001$ \\
Male & $305(37.6 \%)$ & $25(3.1 \%)$ & $32(3.9 \%)$ & $41(5.1 \%)$ & $207(25.5 \%)$ & \\
Female & $501(61.8 \%)$ & $32(3.9 \%)$ & $18(2.2 \%)$ & $51(6.3 \%)$ & $400(49.3 \%)$ & \\
Rather not say & $5(0.6 \%)$ & $0(0.0 \%)$ & $1(0.1 \%)$ & $1(0.1 \%)$ & $3(0.4 \%)$ & \\
Classification & & & & & & .521 \\
Freshmen & $116(14.3 \%)$ & $4(0.5 \%)$ & $9(1.1 \%)$ & $10(1.2 \%)$ & $93(11.5 \%)$ & \\
Sophomore & $105(12.9 \%)$ & $8(1.0 \%)$ & $4(0.5 \%)$ & $14(1.7 \%)$ & $79(9.7 \%)$ & \\
Junior & $168(20.7 \%)$ & $13(1.6 \%)$ & $15(1.8 \%)$ & $18(2.2 \%)$ & $122(15 \%)$ & \\
Senior & $423(52.1 \%)$ & $32(3.9 \%)$ & $23(2.8 \%)$ & $51(6.3 \%)$ & $317(39 \%)$ & \\
Race/Ethnicity & & & & & & .278 \\
Hispanic/Latino & $110(13.5 \%)$ & $7(0.9 \%)$ & $9(1.1 \%)$ & $14(1.7 \%)$ & $80(9.9 \%)$ & \\
African American & $55(6.8 \%)$ & $4(0.5 \%)$ & $4(0.5 \%)$ & $2(0.2 \%)$ & $45(5.5 \%)$ & \\
Native American & $4(0.5 \%)$ & $1(0.1 \%)$ & $0(0.0 \%)$ & $1(0.1 \%)$ & $2(0.2 \%)$ & \\
Asian/Pacific Islander & $98(12.1 \%)$ & $12(1.5 \%)$ & $7(0.9 \%)$ & $12(1.5 \%)$ & $67(8.3 \%)$ & \\
Non-Hispanic White & $504(62.1 \%)$ & $31(3.8 \%)$ & $29(3.6 \%)$ & $55(6.8 \%)$ & $389(47.9 \%)$ & \\
Other & $41(5.0 \%)$ & $2(0.2 \%)$ & $2(0.2 \%)$ & $9(1.1 \%)$ & $28(3.4 \%)$ & \\
\hline
\end{tabular}

Chi-square tests of association for group comparisons 
Table 2 Demographic characteristics mean scores and standard deviation for knowledge, GSE, PHQ, GAD, and GPA $(\mathrm{n}=811)$

\begin{tabular}{llllll}
\hline Characteristic & Knowledge & Self-efficacy & Depression & Anxiety & GPA \\
\hline Gender & & & & & \\
Male & $28.71(4.96)^{*}$ & $31.89(6.02)$ & $12.83(4.77)$ & $10.41(4.33)$ & $3.42(0.37)$ \\
Female & $32.09(4.50)^{*}$ & $31.52(5.15)$ & $13.44(4.76)$ & $11.42(4.37)$ & $3.51(0.36)$ \\
Rather not say & $32.20(3.77)$ & $30.20(5.85)$ & $16.80(6.38)$ & $14.00(6.36)$ & $3.32(0.54)$ \\
Classification & & & & & \\
Freshmen & $31.12(5.15)$ & $32.07(4.81)$ & $13.69(5.73)$ & $11.10(4.72)$ & $3.53(0.35)$ \\
Sophomore & $31.12(4.91)$ & $31.26(5.80)$ & $13.61(4.26)$ & $11.30(4.03)$ & $3.41(0.45)$ \\
Junior & $30.41(5.60)$ & $31.10(5.33)$ & $13.76(5.28)$ & $11.38(4.88)$ & $3.46(0.39)$ \\
Senior & $30.82(5.04)$ & $31.85(5.65)$ & $12.80(4.37)$ & $10.87(4.20)$ & $3.48(0.34)$ \\
Race/Ethnicity & & & & & \\
Hispanic/Latino & $31.18(4.9)$ & $31.51(5.53)$ & $13.94(5.14)$ & $11.51(4.68)$ & $3.33(0.47)$ \\
African American & $30.24(4.65)$ & $30.49(5.55)$ & $14.20(5.24)$ & $11.36(4.63)$ & $3.27(0.38)$ \\
Native American & $30.50(5.45)$ & $32.50(3.79)$ & $13.00(0.82)$ & $12.50(3.0)$ & $3.51(0.51)$ \\
Asian/Pacific Islander & $29.79(4.65)$ & $30.19(6.07)$ & $14.43(5.53)$ & $11.31(4.05)$ & $3.42(0.37)$ \\
Non-Hispanic White & $31.13(4.52)$ & $32.20(5.26)$ & $12.66(4.44)$ & $10.83(4.37)$ & $3.54(0.32)$ \\
Other & $29.59(4.52)$ & $30.20(5.96)$ & $14.12(4.64)$ & $11.63(4.56)$ & $3.34(0.33)$ \\
\hline
\end{tabular}

$G S E$ general self-efficacy scale, $P H Q$ patient health questionnaire, $G A D$ generalized anxiety disorder scale, $G P A$ grade point average

$* \mathrm{p}<0.01$

Table 3 Mean, standard deviation, and bivariate results for knowledge, GSE, PHQ, GAD, and GPA compared to e-cigarette use frequency

\begin{tabular}{|c|c|c|c|c|c|}
\hline Characteristic & Every day $(n=57)$ & Somedays $(n=51)$ & Rarely $(n=93)$ & $\operatorname{Never}(n=611)$ & P-value \\
\hline Knowledge (mean, SD) & $26.61(4.77)$ & $27.33(4.22)$ & $27.70(3.09)$ & $28.66(3.30)$ & $>.001$ \\
\hline Self-efficacy (mean, SD) & $29.02(8.87)$ & $32.18(4.66)$ & $31.67(5.73)$ & $31.85(5.04)$ & .002 \\
\hline Depression (mean, SD) & $13.63(5.03)$ & $14.20(4.54)$ & $13.87(5.40)$ & $13.01(4.67)$ & $.040^{*}$ \\
\hline Anxiety (mean, SD) & $11.39(4.43)$ & $11.67(4.69)$ & $11.60(4.39)$ & $10.90(4.37)$ & .319 \\
\hline GPA (mean, SD) & $3.31(0.38)$ & $3.41(0.33)$ & $3.43(0.33)$ & $3.50(0.37)$ & .001 \\
\hline
\end{tabular}

ANOVA one-way analysis used to compare means

GSE general self-efficacy scale, $P H Q$ patient health questionnaire, GAD generalized anxiety disorder scale, GPA grade point average

*Kruskal-Wallis test for independent samples used due to violation of normalcy

$(\mathrm{M}=13.01, \mathrm{SD}=4.67)$ was significantly lower than somedays users $(M=14.20, S D=4.54)$. Significant differences in depression scores between other e-cigarette frequency categories were not seen. ANOVA analysis indicate a significant relationship between GPA and e-cigarette use frequency $[\mathrm{F}(3,808)=5.49, \mathrm{p}<.001]$. Post hoc comparisons using Hochberg's GT2 test indicate that the average GPA for everyday users $(\mathrm{M}=3.31, \mathrm{SD}=.38)$ was significantly lower than never users $(\mathrm{M}=3.50, \mathrm{SD}=0.37)$.

\section{Discussion}

The current study was designed to assess the associations between outcomes of interest-knowledge of possible negative health effects of e-cigarette use, self-efficacy, anxiety, depression, and academic performance—and e-cigarette use in a sample of college students. Approximately a fourth of respondents (24.8\%) reported e-cigarette use which is consistent with previous research regarding e-cigarette prevalence among college students $[12,15,21]$. Of the variables assessed in this study, significant relationships were seen between knowledge, self-efficacy, depression, and academic performance with e-cigarette use. No significant relationship between anxiety and e-cigarette use was seen (Table 3).

Previous research studies indicate knowledge of risks associated with e-cigarette use to be an indicator of the likelihood to use e-cigarettes $[5,34]$ which was supported by our findings. Significant differences were noted in knowledge scores for everyday users when compared to never users and someday users. This indicates that lower levels of knowledge about the possible negative health outcomes of e-cigarette use significantly correlates to more frequent e-cigarette use. No significant difference was seen 
between rare users and never users indicating that knowledge may not decrease the likelihood of an individual trying e-cigarettes but does influence continued, habitual e-cigarette use behaviors.

High self-efficacy has been established in the literature as a contributory factor to quitting e-cigarette behavior $[7,27]$. However, the influence of low self-efficacy on the frequency of e-cigarette use has not been previously explored. The current study revealed a significant relationship between self-efficacy and all categories of e-cigarette use frequency. High self-efficacy was associated with less frequent and never use of e-cigarettes and low self-efficacy was associated with more frequency e-cigarette use, implying that self-efficacy influences frequency of e-cigarette use.

Strong associations between depression and e-cigarette use have been established by several studies $[2,7,8]$ and our results support the findings of those studies. Our results indicate a significant difference between depression scores for never users when compared to somedays users, indicating rates of depression symptoms tend to be higher among somedays users. A significant difference was not seen between depression scores of everyday users when compared to never users which could be due to the relatively small number of everyday users present in the sample.

Studies have found a relationship between anxiety symptoms and the use of combustible cigarettes [24, 25]. While no significant relationship between anxiety and e-cigarette use was found in our current study, anxiety scores were slightly lower for never users $(\mathrm{M}=10.90, \mathrm{SD}=4.37)$ than for everyday users $(\mathrm{M}=11.39, \mathrm{SD}=4.43)$. The lack of significance could be due to a small number of individuals in the sample meeting the criteria for generalized anxiety disorder. It is possible that participants still use e-cigarettes as a coping mechanism for dealing with feelings of stress and anxiety without meeting the criteria for an anxiety disorder. As research into e-cigarette use progresses, a relationship between anxiety and e-cigarette use may emerge. It is important to understand any possible underlying motivations for e-cigarette use, especially those pertaining to selfmedication of mental illness, in order to effectively reduce e-cigarette use.

The results of our study indicate a significant relationship between academic performance, as assessed using self-reported cumulative GPA, and e-cigarette use. A significantly lower GPA was seen in everyday users when compared to never users. Due to the nature of the cross-sectional survey, the directionality of this relationship could not be determined; therefore, it cannot be discerned whether those with lower academic performance are more likely to use e-cigarettes or if using e-cigarettes in some way leads to a decrease in academic performance.

\section{Limitations}

Cross-sectional surveys are inherently prone to response bias, selection bias, and sample bias. Response bias may have been present as participants were enrolled in a healthbased class and may have felt uncomfortable providing information regarding e-cigarette use in such an environment. Selection bias is seen in the use of a convenience sample. Although the demographic characteristics of the sample population closely mirror those of the university population, sample bias may still be present due to the high proportion of public health students present in the sample population. Furthermore, temporality cannot be determined with the use of a cross-sectional survey. Because the study was conducted on a Tobacco-free, smoke-free, and religious campus, it is possible that e-cigarette use is lower among students in the sample population than would be seen at colleges that lack these stipulations.

\section{Implications}

Notwithstanding the limitations, the findings from our study have strong public health implications. We found approximately one-fourth of the participants are exposed to the harmful effects of e-cigarettes and lack of knowledge or misinformation regarding possible long-term negative health effects of e-cigarette use was correlated with increased e-cigarette use. Public health professionals should focus on education as a tool to reduce the prevalence of e-cigarette use among college students. Additionally, a relationship between low self-efficacy and increased frequency of e-cigarette use was found, indicating that along with providing accurate information, public health workers should focus on increasing self-efficacy. Lastly, emphasizing the importance of healthy outlets for managing stress, anxiety, and depression symptoms could help reduce the use of e-cigarettes as a coping mechanism.

\section{Recommendations}

Future research pertaining to e-cigarette use among college students is needed. Replicating this study at other colleges, specifically colleges without religious affiliations or tobacco-free, smoke-free campuses, could help further illuminate the relationships between e-cigarette use and the target variables. Additionally, future research on the relationship between anxiety and e-cigarette use is needed. Lastly, research focusing on the relationship between 
sustained e-cigarette use and susceptibility to respiratory diseases would be beneficial.

In conclusion, our study findings underscore that e-cigarette use continues to be a public health menace and college students are susceptible to engaging in this harmful behavior. Modifiable factors such as self-efficacy, knowledge, and depression are associated with e-cigarette use. Future intervention can target those modifiable factors to reduce e-cigarette use among college students.

Funding No funding.

\section{Compliance with Ethical Standards}

Conflict of interest The authors do not have any conflict of interest to declare.

Ethical Approval All procedures performed in the study were in accordance with the ethical standards of the institutional research committee and the 1964 Declaration of Helsinki and its later amendments. This article does not contain any studies with animals performed by any of the authors. Permission was obtained from the University's Institutional Review Board (IRB) prior to the beginning of the study.

\section{References}

1. Bacon, D. R., \& Bean, B. (2006). GPA in research studies: An invaluable but neglected opportunity. Journal of Marketing Education, 28(1), 35-42.

2. Bandiera, F. C., Loukas, A., Li, X., Wilkinson, A. V., \& Perry, C. L. (2017). Depressive symptoms predict current e-cigarette use among college students in Texas. Nicotine \& Tobacco Research, 19(9), 1102-1106.

3. Begdache, L., Kianhehr, H., Sabounchi, N., Marszalek, A., \& Doman, N. (2019). Principal component regression of academic performance, substance use, and sleep quality in relation to risk of anxiety and depression in young adults. Trends in Neuroscience and Education., 15, 29-37.

4. Brake, S. J., Barnsley, K., Lu, W., McAlinden, K. D., Eapen, M. S., \& Sohal, S. S. (2020). Smoking upregulates angiotensin-converting enzyme-2 receptor: A potential adhesion site for novel coronavirus SARS-CoV-2 (Covid-19). Journal of Clinical Medicine, 9(3), 841. https://doi.org/10.3390/jcm9030841.

5. Case, K., Crook, B., Lazard, A., \& Mackert, M. (2016). Formative research to identify perceptions of e-cigarettes in college students: Implications for future health communication campaigns. Journal of American College Health, 64(5), 380-389.

6. Cassady, J. C. (2001). Self-reported GPA and SAT: A methodological note. Practical Assessment, Research \& Evaluation, 7(7), 2000-2001.

7. Chan, G., Morphett, K., Gartner, C., Leung, J., Yong, H. H., Hall, W., et al. (2019). Predicting vaping uptake, vaping frequency and ongoing vaping among daily smokers using longitudinal data from the International Tobacco Control (ITC) Four Country Surveys. Addiction, 114, 61-70.

8. Chaiton, M., Cohen, J. E., Rehm, J., Abdulle, M., \& O'Loughlin, J. (2015). Confounders or intermediate variables? Testing mechanisms for the relationship between depression and smoking in a longitudinal cohort study. Addictive Behaviors, 42, 154-161.
9. Coleman, B. N., Apelberg, B. J., Ambrose, B. K., Green, K. M., Choiniere, C. J., Bunnell, R., et al. (2015). Association between electronic cigarette use and openness to cigarette smoking among US young adults. Nicotine and Tobacco Research, 17(2), 212-218.

10. Copeland, A. L., Peltier, M. R., \& Waldo, K. (2017). Perceived risk and benefits of e-cigarette use among college students. Addictive Behaviors, 71, 31-37.

11. Counotte, D. S., Spijker, S., van de Burgwal, L. H., Hogenboom, F., Schoffelmeer, A. N. M., de Vries, T. J., et al. (2009). Longlasting cognitive deficits resulting from adolescent nicotine exposure in rats. Neuropsychopharmacology, 34(2), 299-306.

12. Delnevo, C. D., Giovenco, D. P., Steinberg, M. B., Villanti, A. C., Pearson, J. L., Niaura, R. S., et al. (2016). Patterns of electronic cigarette use among adults in the United States. Nicotine \& Tobacco Research, 18, 715-719.

13. Fountain, S. B., Rowan, J. D., Kelley, B. M., Willey, A. R., \& Nolley, E. P. (2008). Adolescent exposure to nicotine impairs adult serial pattern learning in rats. Experimental Brain Research, 187(4), 651-656.

14. Grana, R. A., \& Ling, P. M. (2014). "Smoking revolution": A content analysis of electronic cigarette retail websites. American Journal of Preventive Medicine, 46(4), 395-403.

15. Grant, J. E., Lust, K., Fridberg, D. J., King, A. C., \& Chamberlain, S. R. (2019). E-cigarette use (vaping) is associated with illicit drug use, mental health problems, and impulsivity in university students. Annals of Clinical Psychiatry: Official Journal of the American Academy of Clinical Psychiatrists, 31(1), 27.

16. Hefner, K. R., Sollazzo, A., Mullaney, S., Coker, K. L., \& Sofuoglu, M. (2019). E-cigarettes, alcohol use, and mental health: Use and perceptions of e-cigarettes among college students, by alcohol use and mental health status. Addictive Behaviors, 91, 12-20.

17. Iiguez, S. D., Warren, B. L., Parise, E. M., Alcantara, L. F., Schuh, B., Maffeo, M. L., et al. (2009). Nicotine exposure during adolescence induces a depression-like state in adulthood. Neuropsychopharmacology, 34(6), 1609-1624.

18. Kang, H. (2013). The prevention and handling of the missing data. Korean Journal of Anesthesiology, 64(5), 402-406. https://doi. org/10.4097/kjae.2013.64.5.402.

19. Kosmider, L., Sobczak, A., Fik, M., Knysak, J., Zaciera, M., Kurek, J., et al. (2014). Carbonyl compounds in electronic cigarette vapors: Effects of nicotine solvent and battery output voltage. Nicotine \& Tobacco Research, 16(10), 1319-1326.

20. Kroenke, K., Spitzer, R. L., \& Williams, J. B. (2001). The PHQ-9: validity of a brief depression severity measure. Journal of General Internal Medicine, 16(9), 606-613.

21. Lanza, H. I., \& Teeter, H. (2018). Electronic nicotine delivery systems (e-cigarette/vape) use and co-occurring health-risk behaviors among an ethnically diverse sample of young adults. Substance Use \& Misuse, 53(1), 154-161.

22. Lechner, W. V., Janssen, T., Kahler, C. W., Audrain-McGovern, J., \& Leventhal, A. M. (2017). Bi-directional associations of electronic and combustible cigarette use onset patterns with depressive symptoms in adolescents. Preventive Medicine, 96, 73-78.

23. Luo, C., Zheng, X., Zeng, D. D., \& Leischow, S. (2014). Portrayal of electronic cigarettes on YouTube. BMC Public Health, 14(1), $1028-1028$

24. Mojtabai, R., \& Crum, R. M. (2013). Cigarette smoking and onset of mood and anxiety disorders. American Journal of Public Health, 103(9), 1656-1665.

25. Mykletun, A., Overland, S., Aar $\varnothing$, L. E., Liabø, H. M., \& Stewart, R. (2008). Smoking in relation to anxiety and depression: evidence from a large population survey: The HUNT study. European Psychiatry, 23(2), 77-84.

26. Obisesan, O. H., Mirbolouk, M., Osei, A. D., Orimoloye, O. A., Uddin, S. I., Dzaye, O., et al. (2019). Association between 
e-cigarette use and depression in the behavioral risk factor surveillance system, 2016-2017. JAMA Network Open, 2(12), e1916800.

27. Pokhrel, P., Fagan, P., Little, M. A., Kawamoto, C. T., \& Herzog, T. A. (2013). Smokers who try e-cigarettes to quit smoking: Findings from a multiethnic study in Hawaii. American Journal of Public Health, 103(9), e57-e62.

28. Schoenborn, C., \& Gindi, R. (2016). Cigarette smoking status among current adult E-cigarette users, by age group-national health interview survey, united states, 2015. MMWR-Morbidity and Mortality Weekly Report, 65(42), 1177-1177.

29. Schwarzer, R., \& Jerusalem, M. (1995). Generalized self-Efficacy scale. In J. Weinman, S. Wright \& M. Johnston (Eds.), Measures in health psychology: A user's portfolio. Causal and control beliefs (pp. 35-37). Windsor, UK: NFER-Nelson.

30. Spitzer, R. L., Kroenke, K., Williams, J. B., \& Löwe, B. (2006). A brief measure for assessing generalized anxiety disorder: The GAD-7. Archives of Internal Medicine, 166(10), 1092-1097.

31. The National Center on Addiction and Substance Abuse. (2008). Beyond cigarettes: The risks of non-cigarette nicotine products and implications for tobacco control. The National Center on Addiction and Substance Abuse. https://www.centeronaddicti on.org/.
32. U.S. Department of Health and Human Services. (2016). E-cigarette use among youth and young adults. A report of the Surgeon General. Atlanta, GA: US Department of Health and Human Services, Centers for Disease Control and Prevention, National Center for Chronic Disease Prevention and Health Promotion, Office on Smoking and Health.

33. Wallace, L. N., \& Roache, M. J. (2018). Vaping in context: Links among E-cigarette use, social status, and peer influence for college students. Journal of Drug Education, 48(102), 36-53.

34. Wiseman, K. P., Margolis, K. A., Bernat, J. K., \& Grana, R. A. (2019). The association between perceived e-cigarette and nicotine addictiveness, information-seeking, and e-cigarette trial among US adults. Preventive Medicine, 118, 66-72.

Publisher's Note Springer Nature remains neutral with regard to jurisdictional claims in published maps and institutional affiliations. 